\title{
AN ELEMENTARY COUNTEREXAMPLE TO THE OPEN MAPPING PRINCIPLE FOR BILINEAR MAPS
}

\author{
CHARIES HOROWITZ
}

\begin{abstract}
In [2], Rudin asked whether a continuous bilinear map from the product of two Banach spaces onto a Banach space must be open at the origin; i.e., whether under such a map the image of every neighborhood of zero must contain a neighborhood of zero. Recently, Cohen [1] showed that the answer to the general question was in the negative. However, his counterexample was somewhat involved and left the issue unresolved for bilinear maps on Hilbert spaces. The purpose of this note is to show that the open mapping principle for bilinear maps, as described above, fails even in the finite dimensional case.
\end{abstract}

Specifically, we consider a mapping $T: \mathbf{C}^{3} \times \mathbf{C}^{3} \rightarrow \mathbf{C}^{4}$, defined as follows:

$$
T(x, y)=\left(x_{1} y_{1}, x_{1} y_{2}, x_{1} y_{3}+x_{3} y_{1}+x_{2} y_{2}, x_{3} y_{2}+x_{2} y_{1}\right),
$$

where, e.g., $x=\left(x_{1}, x_{2}, x_{3}\right)$. Clearly $T$ is bilinear and continuous. We show that $T$ is surjective but not open at the origin.

To verify surjectivity, let $z=\left(z_{1}, z_{2}, z_{3}, z_{4}\right) \in \mathbf{C}^{4}$ be given. If $z_{1}=$ $z_{2}=0$, choose $x=\left(0, z_{4}, z_{3}\right), y=(1,0,0)$, and, then, $T(x, y) \ldots z$. On the other hand, if, e.g., $z_{1} \neq 0$, let $x=\left(1, z_{4} / z_{1}, 0\right), y=\left(z_{1}, z_{2}, z_{3} \cdots\left(z_{4} z_{2} / z_{1}\right)\right)$, and, again, $T(x, y)=z$. The case $z_{2} \neq 0$ may be handled similarly, and so $T$ is surjective.

It remains to show that $T$ is not open at the origin. To that end, suppose that $T(x, y)=z=(a, a, a, 1)$, where $a$ is a small positive number. From the definition of $T$, we conclude immediately that $y_{1}=y_{2}=a / x_{1}$, $x_{2}+x_{3}=x_{1} / a, y_{3}=(a-1) / x_{1}$, so that $\left(x_{1}+x_{2}+x_{3}\right)\left(y_{1}+y_{2}+y_{3}\right)=\left(x_{1}+x_{1} / a\right)\left(2 a / x_{1}+(a-1) / x_{1}\right)=3 a+2-1 / a$.

Now if $T$ were open at the origin, every bounded subset of $\mathbf{C}^{4}$ could be obtained as the image of a bounded subset of $\mathbf{C}^{3} \times \mathbf{C}^{3}$. Letting $a \rightarrow 0$ in the above calculations, we see that this condition is violated, and so $T$ is not open at the origin.

We remark that the above example goes over unchanged if we replace $\mathbf{C}^{3}$ by $\mathbf{R}^{3}$ and $\mathbf{C}^{4}$ by $\mathbf{R}^{4}$. Moreover, a counterexample in the infinite

Received by the editors December 20, 1974.

AMS (MOS) subject classifications (1970). Primary 15A63, 45A30; Secondary $28 \mathrm{~A} 78$. 
dimensional case is provided by the mapping

$$
T_{1}: \mathbf{C}^{3} \oplus \mathbf{C} \oplus l^{2} \times \mathbf{C}^{3} \oplus l^{2} \rightarrow \mathbf{C}^{4} \oplus l^{2}
$$

defined by $T_{1}\left(x_{1}, x_{2}, x_{3}, y_{1}, y_{2}\right)=\left(T\left(x_{1}, y_{1}\right), x_{2} y_{2}\right)$, where $x_{1}, y_{1} \in \mathbf{C}^{3}$, $x_{3}, y_{2} \in l^{2}, x_{2} \in \mathbf{C}$, and $x_{2} y_{2}$ indicates scalar multiplication.

Finally, we remark that when Rudin asked the question presently under consideration, the application he envisioned was to a symmetric bilinear mapping. For completeness, we include here a counterexample to the open mapping principle in the symmetric case. Indeed, let $T_{2}: \mathbf{C}^{4} \times \mathbf{C}^{4} \rightarrow \mathrm{C}^{5}$ be defined by

$$
\begin{aligned}
T_{2}(x, y)=\left(x_{1} y_{1}, x_{1} y_{2}+x_{2} y_{1}, x_{1} y_{3}+x_{3} y_{1},\right. \\
\left.x_{1} y_{4}+x_{4} y_{1}+x_{2} y_{3}+x_{3} y_{2}, x_{2} y_{2}+x_{3} y_{3}\right) .
\end{aligned}
$$

It is not hard to show that $T_{2}$ maps $\mathbf{C}^{4} \times \mathbf{C}^{4}$ onto $\mathbf{C}^{5}$ (although the corresponding mapping on $\mathbf{R}^{4} \times \mathbf{R}^{4}$ is not surjective), but that on the subspaces $x_{1}=0$ and $y_{1}=0, T_{2}$ essentially reduces to our original $T$, From this it follows that the preimages under $T_{2}$ of points of the form $(0, a, a$, $a, 1)$ must "go to infinity" as $a \rightarrow 0$.

\section{REFERENCES}

1. P. J. Cohen, A counterexample to the closed graph theorem for bilinear maps, J. Functional Analysis 16 (1974), 235-239.

2. W. Rudin, Function theory in polydiscs, Benjamin, New York, 1969. MR $41 \# 501$.

DEP ARTMENT OF MATHEMATICS, UNIVERSITY OF WISCONSIN, MADISON, WISCONSIN 53706 